\title{
ARTHROSCOPIC REPAIR OF SMALL AND MEDIUM TEARS OF THE SUPRASPINATUS MUSCLE TENDON: EVALUATION OF THE CLINICAL AND FUNCTIONAL OUTCOMES AFTER TWO YEARS OF FOLLOW-UP
}

Roberto Yukio Ikemoto', Joel Murachovsky², Luís Gustavo Prata Nascimento ${ }^{3}$, Rogério Serpone Bueno ${ }^{3}$, Luis Henrique Almeida ${ }^{4}$, Eric Strose $^{3}$, Marcello Teixeira Castiglia ${ }^{5}$

\begin{abstract}
Objective: To evaluate the clinical and functional outcomes from arthroscopic repairs on small and medium-sized tears of the supraspinatus muscle tendon. Methods: 129 cases of isolated small and medium tears of the supraspinatus muscle tendon were evaluated retrospectively. The average duration of pain was 29 months. The average joint range of motion comprised active elevation of $136^{\circ}$, lateral rotation of $58^{\circ}$ and medial rotation at $\mathrm{T} 12$ level; and the preoperative functional UCLA score averaged 17 points. In all the cases, complete repair could be achieved. Results: The average score on the UCLA functional scale in the postoperative period was 32 points. The average length of follow-up was 39 months. Seventy-five cases (58\%) had excellent results and 42 (32\%) had good results. The average final active elevation was $156^{\circ}$
\end{abstract}

\section{INTRODUCTION}

Injuries to the tendons of the rotator cuff are among the commonest causes of pain and functional impotence in adults' shoulders ${ }^{(1,2)}$. These injuries may occur in one of the tendons in isolation, and the commonest of these is injuries of the supraspinatus tendon $^{(3)}$, which is inserted into the greater tubercle, is around $16 \mathrm{~mm}$ in length and is microscopically subdivided into five distinct layers ${ }^{(4,5)}$.

These injuries can be classified as partial or com- with an average gain of $20^{\circ}$, and the average final lateral rotation was $57^{\circ}$ with an average gain of $9^{\circ}$. Both of these were statistically significant $(\mathrm{P}<0.05)$. The patients who underwent tenotomy of the long head of the biceps (LHB), with or without tenodesis, did not present statistically inferior functional outcomes, in comparison with the patients who only underwent decompression and lesion repair $(\mathrm{P}=1.00)$. Fourteen cases $(10.8 \%)$ presented complications during the postoperative period. Six (4.6\%) developed adhesive capsulitis and four $(3.1 \%)$ presented re-rupture of the tendon, proven by means of magnetic resonance imaging. Conclusions: Arthroscopic repair of small and medium tears of the supraspinatus muscle tendon provided a functional clinical improvement, with good and excellent results in $90 \%$ of the cases.

Keywords - Shoulder; Arthroscopy; Rotator Cuff/injuries plete. Although it has been reported in the literature that these injuries are common among patients over the age of 60 years, such patients are in many cases asymptomatic $^{(3,6)}$.

Surgical treatment for rotator cuff injuries is indicated if conservative treatment fails ${ }^{(3,7,8)}$. Arthroscopic repair of the injury is one of the possibilities and, with the evolution of this technique, it is becoming increasingly popular ${ }^{(1,3,9)}$.

Arthroscopic repairs to small and medium-sized

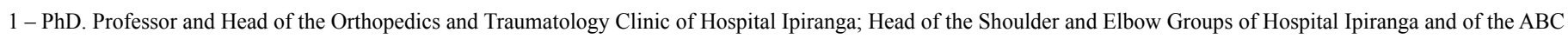
Medical School, Santo André, SP, Brazil.

2 - PhD. Professor and Attending Physician in the Shoulder and Elbow Group, ABC Medical School, Santo André, SP, Brazil.

3 - MSc in Orthopedics; Attending Physician in the Shoulder and Elbow Group, ABC Medical School, Santo André, SP, Brazil.

4 - Attending Physician in the Shoulder and Elbow Group, ABC Medical School, Santo André, SP, Brazil.

5 - Trainee Physician in Shoulder and Elbow Surgery, ABC Medical School, Santo André, SP, and Hospital Ipiranga, São Paulo, SP, Brazil.

Work performed in the Discipline of Diseases of the Locomotor Apparatus, ABC Medical School. Director: Prof. Dr. Carlo Milani.

Correspondence: Rua Traipu 1269, Pacaembu, 01235-000 São Paulo, SP. E-mail: jd.mura@uol.com.br

Work received for publication: May 25, 2011; accepted for publication: August 31, 2011.

The authors declare that there was no conflict of interest in conducting this work 
injuries to the tendon of the supraspinatus muscle have excellent results, and $84 \%$ of the cases maintain the integrity of the repair ${ }^{(10)}$. Integrity of the repair leads to better function, mobility and strength, in comparison with cases that present renewed tearing of the tendon of the supraspinatus muscle $\mathrm{e}^{(8,11)}$.

The aim of this study was to assess the clinical and functional results from arthroscopic repairs to small and medium-sized injuries to the tendon of the supraspinatus muscle, with a minimum follow-up of two years.

\section{SAMPLE AND METHODS}

Between February 2002 and July 2008, the Shoulder and Elbow Group of the Department of Orthopedics and Traumatology of the ABC Medical School treated 216 patients with isolated injuries of the tendon of the supraspinatus muscle, arthroscopically. The case inclusion criteria that we used were that the patients should present isolated injuries to this tendon and should not have had any previous surgical treatment, and that the tears should be classified as small or medium (lesions of less than $3 \mathrm{~cm}$, in accordance with DeOrio and Cofield ${ }^{(12)}$ and Gartsman $\left.{ }^{(13)}\right)$, with confirmation by means of magnetic resonance imaging. Patients who had undergone previous surgical procedures were not included, nor were cases with less than two years of postoperative follow-up. Eighty-seven patients were excluded because they were lost from the follow-up. Thus, 129 cases were reassessed retrospectively, among which 48 were male and 81 were female, with a mean age of 55 years, ranging from 38 to 80 years; the dominant limb was affected in 94 cases. The pain had begun insidiously in 102 patients and after an episode of trauma in 27. The length of time with pain resulting from the injury to the tendon of the supraspinatus muscle, until the surgery was performed, ranged from one to 204 months (mean of 29 months). These patients presented mean active elevation of $136^{\circ}$ (ranging from 60 to $160^{\circ}$ ), measured using a manual goniometer ${ }^{(14)}$. The functional assessment performed before the operation according to the scale of the University of California, Los Angeles (UCLA) ${ }^{15)}$, ranged from 8 to 31 points (mean of 17 points).

All the cases underwent arthroscopic repair of the lesion in the deckchair position. Suturing was performed using metal anchors and ultra-resistant thread. In
62 of these cases (48\%), we observed abnormalities of the tendon of the long head of the brachial biceps muscle (LHB tendon), which included complete absence of the tendon, partial lesions, thickening and instability in the bicipital sulcus. In all the cases in which the tendon was not completely torn (61 cases), tenotomy was performed. In 14 of these, this was done in association with tenodesis, by means of a suturing technique with an anchor or an interference screw.

Acromioplasty was performed routinely in all the patients. In the cases with symptomatic arthrosis of the acromioclavicular joint, we resected the distal extremity of the clavicle, by means of an arthroscopic technique. All the lesions were measured before the operation, by means of magnetic resonance, and during the operation with the aid of calipers measuring in millimeters.

Complete repair of the lesion was possible in all the cases. In 24 cases, tendon-to-tendon stitches had to be used: in 10 of these, with a single stitch; in 11 cases, two stitches; and in three cases, three stitches. The mean number of anchors used was two (ranging from one to three).

During the postoperative period, all the patients were immobilized using a sling. For the first six weeks, the patients were allowed to make flexionextension movements of the elbow and passive lateral rotation up to the pain threshold. After this period, the immobilization was withdrawn and the patients started to do passive and assisted active stretching exercises in order to gain active range of motion of the shoulder. After the fourth postoperative month, muscle strengthening exercises for the rotator cuff and scapular belt were started.

The final functional results were evaluated in accordance with the UCLA functional scale. For the statistical analysis, the LogXact 6.2 (LogXact, Cytel Software, Cambridge, USA) and Minitab v13 (Minitab, Pennsylvania, USA) computer software was used. By means of the paired Student's t test, we sought to assess whether there was any statistically significant difference in the UCLA functional scores regarding pre and postoperative mobility. We used univariate exact binary logistic regression models to investigate whether there was any relationship between unsatisfactory results and longer duration of preoperative pain or between unsatisfactory results and performing tenotomy procedures, with or without tenodesis of the LHB tendon. 


\section{RESULTS}

The minimum length of follow-up was 24 months and the maximum was 72 months (mean of 39 months). According to the UCLA classification system, 75 cases $(58 \%)$ had excellent results (Figure 1), 42 cases (32\%) good, $10(7.7 \%)$ fair and two $(2.3 \%)$ poor. Thus, we found that $90 \%$ of the functional results were satisfactory, with a mean score of 32 points (ranging from 13 to 35 points). According to the paired Student's t test, there was a statistically significant functional improvement $(\mathrm{P}<0.05)$, compared with the preoperative assessment (Table 1).

The mean final active elevation was $156^{\circ}$ (ranging from 40 to $160^{\circ}$ ), with a mean gain of $20^{\circ}$. The mean final lateral rotation was $57^{\circ}$ (ranging from 10 to $80^{\circ}$ ), with a mean gain of $9^{\circ}$. There was a mean gain in medial rotation of three vertebral levels. According to the paired Student's $t$ test, all these results were statistically significant $(\mathrm{P}<0.05)$ (Table 2$)$.

The patients who underwent other procedures, such as resection of the lateral extremity of the clavicle, tenotomy and tenodesis of the LHB tendon, did not present statistically inferior functional results, in relation to those who only underwent acromioplasty and lesion repair $(\mathrm{P}=1.00)$.

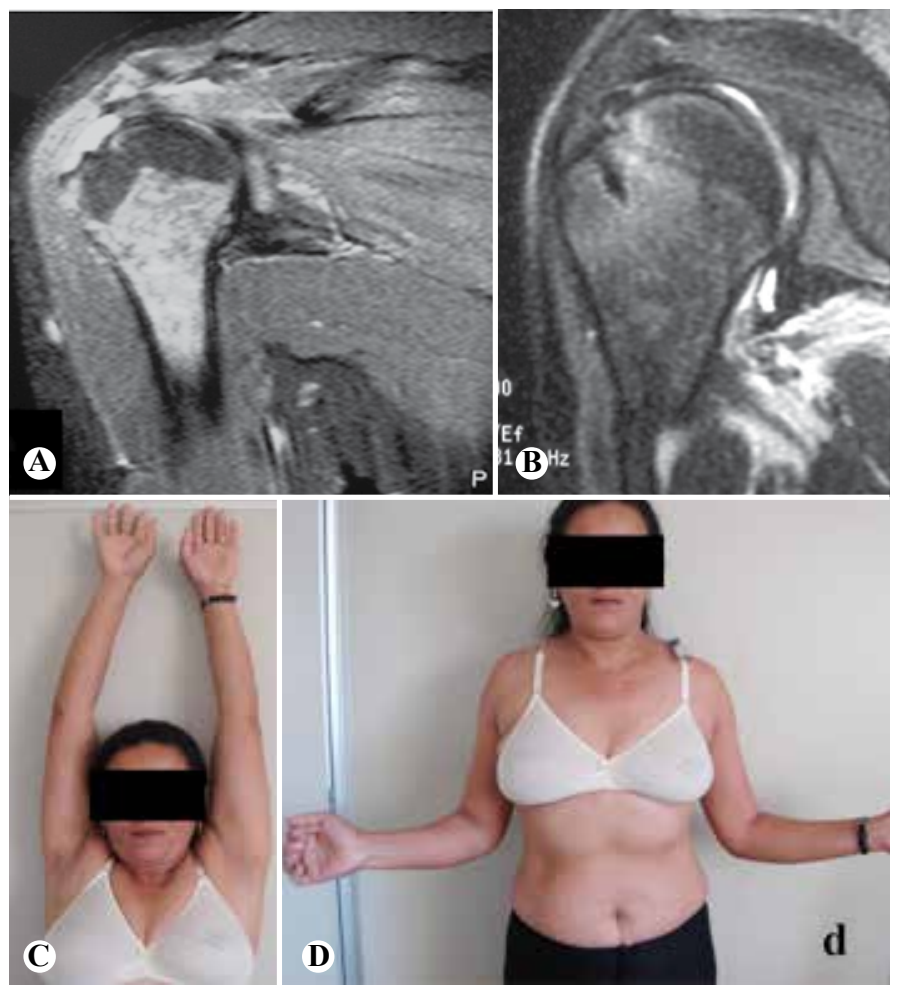

Figure 1 - (A) Preoperative magnetic resonance imaging, showing complete tearing of the tendon of the supraspinatus muscle; (B) postoperative magnetic resonance imaging, showing healing of the tendon; $(C)$ and $(D)$ elevation and lateral rotation one year after the operation, with UCLA $=35$.
Table 1 - Results according to UCLA assessment criteria.

\begin{tabular}{c|c|c}
\hline \multirow{2}{*}{ Satisfactory } & Results & Percentage \\
\cline { 2 - 3 } & 75 excellent & $58 \%$ \\
\hline \multirow{2}{*}{ Unsatisfactory } & 42 good & $32 \%$ \\
\cline { 2 - 3 } & 10 fair & $7.7 \%$ \\
\hline Total & 2 poor & $2.3 \%$ \\
\hline
\end{tabular}

Source: Discipline of Diseases of the Locomotor Apparatus, ABC Medical School.

Table 2 - Mean pre and postoperative mobility.

\begin{tabular}{c|c|c}
\hline Mobility & Preoperative & Postoperative \\
\hline Elevation & $136^{\circ}$ & $157^{\circ}-\mathrm{P}<0.05$ \\
\hline Lateral rotation & $48^{\circ}$ & $57^{\circ}-\mathrm{P}<0.05$ \\
\hline Medial rotation & $\mathrm{T} 12$ & $\mathrm{~T} 9-\mathrm{P}<0.05$ \\
\hline
\end{tabular}

Source: Discipline of Diseases of the Locomotor Apparatus, ABC Medical School.

In our study, there was no statistically significant difference in the final result, with regard to the length of time with pain in relation to any of the variables studied except for the measurements of final medial rotation, which were shown to be greater in cases with shorted length of time with pain, and this was statistically significant $(\mathrm{p}=0.02)$.

Fourteen cases $(10.8 \%)$ presented postoperative complications. Two cases had intercurrences during the immediate postoperative period (post-blockage pleural effusion and neuropraxia of the median nerve), which presented complete resolution by the $12^{\text {th }}$ week after the operation and satisfactory final results (good or excellent results, according to the UCLA scale). Two cases were individuals with work-related problems and, despite all the efforts, they had poor results (UCLA 13 and 15). Six patients (4.6\%) developed adhesive capsulitis: four of them improved with serial blockage of the suprascapular nerve and achieved a satisfactory final score (UCLA greater than or equal to 29); one case achieved a fair final result (UCLA 23); and one other case had a poor result (UCLA 15). Four cases $(3.1 \%)$ evolved with renewed tearing, and this was confirmed by means of a new magnetic resonance imaging examination that was requested after the operation for patients who continued to have complaints of pain despite the therapeutic measures, or when the clinical improvement was interrupted by the return of pain (in one of these cases). In one of these cases, the lesion was shown to be partial, and the result remained satisfactory (UCLA 28), while the other three presented complete tears that evolved with unsatisfactory or fair results (UCLA 19, 23 and 27), the first two of these underwent revision arthroscopy for repairs after the diagnosis (Table 3 and Figure 2). 
Table 3 - Complications, treatment and final result.

\begin{tabular}{c|c|c}
\hline Complications & Treatment & UCLA \\
\hline AC & SS blockage & 15 \\
\hline AC & SS blockage & 23 \\
\hline AC & SS blockage & 29 \\
\hline AC (3 cases) & SS blockage & 35 \\
\hline Re-RCl without trauma & Reoperation & 19 \\
\hline Re-RCl after trauma & Reoperation & 23 \\
\hline Partial re- $\mathrm{RCl}$ & Expectant & 27 \\
\hline
\end{tabular}

Source: Discipline of Diseases of the Locomotor Apparatus, ABC Medical School.

$\mathrm{AC}=$ adhesive capsulitis; $\mathrm{SS}$ = suprascapular; $\mathrm{Re}-\mathrm{RCI}=$ renewed tearing of rotator cuff injury.

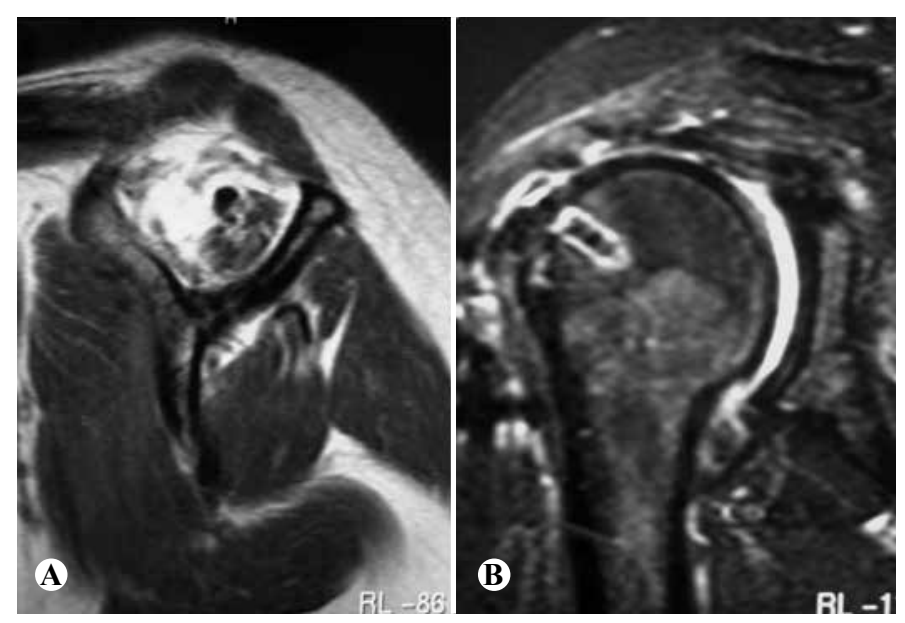

Figure 2 - (A) Preoperative magnetic resonance imaging, showing atrophy of the muscle belly of the supraspinatus; $(B)$ postoperative magnetic resonance imaging, showing renewed tearing of the tendon of the supraspinatus, with UCLA $=10$.

\section{DISCUSSION}

Although there are studies that have shown that there were no differences in the functional results from surgical treatment on the LHB tendon, between different lesion sizes ${ }^{(7,10,16-19)}$, we believe that the chances of renewed tearing become greater with increasing lesion size. This does not necessarily mean that patients will evolve with pain or a poor functional result, but they will at least present diminished strength in the affected limb and some degree of functional limitation, which may change the scoring on any functional scale $\mathrm{s}^{(8,10,16,19)}$.

For this reason, we decided in this study to evaluate and compare the functional results only from cases with small and medium-sized lesions ${ }^{(12,13)}$. In previous studies, it was observed that patients who originally had a lesion of size greater than $3 \mathrm{~cm}$ presented a sevenfold greater chance of evolving with renewed tearing during the follow-up. These patients did not present statistical differences with regard to the issues of pain and functional result, but they showed dimini- shed elevation strength and lateral rotation ${ }^{(19)}$. Other authors identified that cases with extensive lesions presented worse functional results, compared with cases with small and medium-sized lesions ${ }^{(20)}$.

Another study showed that patients whose repairs remained anatomically intact presented greater gains of elevation strength than shown by patients who evolved with renewed tearing, although without any difference in the final results relating to pain and patient satisfaction ${ }^{(16)}$. In cases in which the integrity of the rotator cuff repair was maintained, there was a statistically significant improvement in functional results. Lesions were classified as small if they were less than $1 \mathrm{~cm}$ in size. Out of the 19 cases studied with lesions smaller than $3 \mathrm{~cm}$ in size, integrity was maintained in $84 \%$ of them, thus giving credibility to arthroscopic repair for these types of lesions ${ }^{(10)}$.

In our study, we found that $90 \%$ of the results were satisfactory, among which 58\% were excellent and $32 \%$ were good, according to the UCLA functional scale. This satisfaction index was similar to what was found in the literature ${ }^{(1,7,8,21-26)}$. Although our results were similar to what was found in the literature, two of the four cases that suffered renewed tearing of the supraspinatus (as shown by magnetic resonance imaging) presented unsatisfactory functional results, with complaints of pain and dissatisfaction with the results.

In 1982, Neviaser et $a l^{(23)}$ commented on the relationship between rotator cuff injuries and lesions of the LHB tendon, and other authors have shown an association between such injuries and instability of the LHB tendon ${ }^{(1,3,7,16,17,27,28)}$. In our study, among the 129 cases of rotator cuff injury that underwent operations, $62(48 \%)$ presented abnormalities of the LHB tendon and, except for one case that presented complete tearing of this tendon, all of them underwent tenotomy. In 14 cases, this was done in association with tenodesis of this tendon. The cases in which these procedures were performed presented functional results similar to those in which these procedures were not performed $(\mathrm{P}=1.00)$. This leads us to believe, at least from our study, that performing tenotomy (with or without associated tenodesis) does not impair the final functional result for these patients.

Although Gartsman and O'Connor ${ }^{(29)}$ did not demonstrate any statistically different results in comparing cases of rotator cuff repair with or without associated acromioplasty, we preferred to perform 
acromioplasty in all the cases at our clinic, since we believe that this is a procedure that does not add much time to the surgery and that, in the hands of experienced individuals, it presents low morbidity.

Thus, on average, our cases presented a statistically significant improvement in joint mobility and in the functional result, as confirmed by the paired Student's t test. The mean active elevation achieved by our patients improved from $136^{\circ}$ to $157^{\circ}$. The lateral rotation increased from $48^{\circ}$ to $57^{\circ}$ and the medial rotation showed a mean gain of three vertebral levels. The functional evaluation using the UCLA scale went from an initial mean of 17 points to a score of 32 points at the final assessment.

Regarding the complications observed in our study, six cases $(4.6 \%)$ evolved with adhesive capsulitis and four of them improved satisfactorily with serial blockage of the suprascapular nerve. We also found four cases $(3.1 \%)$ of renewed tearing of the tendon of the supraspinatus muscle, as confirmed by magnetic resonance imaging. One case suffered another trauma during the postoperative period and the other three cases presented spontaneous renewed tearing even though the entire postoperative protocol had been followed properly.

\section{CONCLUSIONS}

Arthroscopic repair on the small and medium-sized injuries to the tendon of the supraspinatus muscle provided clinical and function improvements, such that $90 \%$ of the results were excellent or good.

\section{REFERENCES}

1. Murray TF Jr, Lajtai G, Mileski RM, Snyder SJ. Arthroscopic repair of medium to large full-thickness rotator cuff tears: outcome at 2- to 6-year follow-up. J Shoulder Elbow Surg. 2002;11(1):19-24

2. Walch G, Edwards TB, Boulahia A, Nové-Josserand L, Neyton L, Szabo I. Arthroscopic tenotomy of the long head of the biceps in the treatment of rotator cuff tears: clinical and radiographic results of 307 cases. J Shoulder Elbow Surg. 2005;14(3):238-46

3. Apreleva M, Ozbaydar M, Fitzgibbons PG, Warner JJ. Rotator cuff tears: the effect of the reconstruction method on three-dimensional repair site area. Arthroscopy. 2002;18(5):519-26.

4. Curtis AS, Burbank KM, Tierney JJ, Scheller AD, Curran AR. The insertional footprint of the rotator cuff: an anatomic study. Arthroscopy. 2006;22(6):609.e1.

5. Clark JM, Harryman DT 2nd. Tendons, ligaments, and capsule of the rotator cuff. Gross and microscopic anatomy. J Bone Joint Surg Am. 1992;74(5):713-25.

6. Duralde XA, Bair B. Massive rotator cuff tears: the result of partial rotator cuff repair. J Shoulder Elbow Surg. 2005; 14(2):121-7.

7. Checchia SL, Doneaux PS, Miyazaki AN, Fregonese M, Silva LA, Ishi M, et al. Avaliação dos resultados obtidos na reparação artroscópica das lesões do manguito rotador. Rev Bras Ortop. 2005;40(5):229-38.

8. Wilson F, Hinov V, Adams G. Arthroscopic repair of full-thickness tears of the rotator cuff: 2- to 14-year follow-up. Arthroscopy. 2002;18(2):136-44.

9. Cummins CA, Strickland S, Appleyard RC, Szomor ZL, Marshall J, Murrell GA. Rotator cuff repair with bioabsorbable screws: An in vivo and ex vivo investigation. Arthroscopy. 2003;19(3):239-48.

10. Bishop J, Klepps S, Lo IK, Bird J, Gladstone JN, Flatow EL. Cuff integrity after arthroscopic versus open rotator cuff repair: a prospective study. J Shoulder Elbow Surg. 2006;15(3):290-9

11. Harryman DT 2nd, Mack LA, Wang KY, Jackins SE, Richardson ML, Matsen FA 3rd. Repairs of the rotator cuff. Correlation of functional results with integrity of the cuff. J Bone Joint Surg Am. 1991;73(7):982-9.

12. DeOrio JK, Cofield RH. Results of a second attempt at surgical repair of a failed initial rotator-cuff repair. J Bone Joint Surg Am. 1984; 66(4):563-7.

13. Gartsman GM. Arthroscopic assessment of rotator cuff tear reparability. Arthroscopy. 1996;12(5):546-9.

14. American Academy of Orthopaedics Surgeons. Joint Motion. Method of Measuring and Recording. Chicago: AAOS;1965.

15. Amstutz HC, Sew Hoy AL, Clarke IC. UCLA anatomic total shoulder arthroplasty. Clin Orthop Relat Res. 1981;(155):7-20.
16. Boileau P, Brassart N, Watkinson DJ, Carles M, Hatzidakis AM, Krishnan SG. Arthroscopic repair of full-thickness tears of the supraspinatus: does the tendon really heal? J Bone Joint Surg Am. 2005;87(6):1229-40.

17. Watson EM, Sonnabend DH. Outcome of rotator cuff repair. J Shoulder Elbow Surg. 2002;11(3):201-11.

18. Sugaya H, Maeda K, Matsuki K, Moriishi J. Functional and structural outcome after arthroscopic full-thickness rotator cuff repair: single-row versus dual-row fixation. Arthroscopy. 2005;21(11):1307-16.

19. Verma NN, Dunn W, Adler RS, Cordasco FA, Allen A, MacGillivray J, et al. All-arthroscopic versus mini-open rotator cuff repair: a retrospective review with minimum 2-year follow-up. Arthroscopy. 2006;22(6):587-94.

20. lannotti JP, Bernot MP, Kuhlman JR, Kelley MJ, Williams GR. Postoperative assessment of shoulder function: a prospective study of full-thickness rotator cuff tears. J Shoulder Elbow Surg. 1996;5(6):449-57.

21. Severud EL, Ruotolo C, Abbott DD, Nottage WM. All-arthroscopic versus mini-open rotator cuff repair: A long-term retrospective outcome comparison. Arthroscopy. 2003;19(3):234-8.

22. Rebuzzi E, Coletti N, Schiavetti S, Giusto F. Arthroscopic rotator cuff repair in patients older than 60 years. Arthroscopy. 2005;21(1):48-54.

23. Neviaser TJ, Neviaser RJ, Neviaser JS, Neviaser JS. The four-in-one arthroplasty for the painful arc syndrome. Clin Orthop Relat Res. 1982;(163):107-12.

24. Godinho GG, Souza JMG. Bicalho LA. Reparo das rupturas do man- guito rotador do ombro pela videoartroscopia cirúrgica: técnica. Rev Bras Ortop. 1996;31(4):284-8,

25. Almeida A, Valin MR, Almeida NC, Ferreira R. Avaliação da dor pós-sutura artroscópica do manguito rotador. Rev Bras Ortop. 2006;41(9):341-6.

26. Veado MAC, Almeida Filho IA, Duarte RG, Leitão I. Avaliação funcional do reparo artroscópico das lesões completas do manguito rotador associado a acromioplastia. Rev Bras Ortop. 2008;43(11/12):505-12.

27. Kempf JF, Gleyze P, Bonnomet F, Walch G, Mole D, Frank A, et al. A multicenter study of 210 rotator cuff tears treated by arthroscopic acromioplasty. Arthroscopy. 1999;15(1):56-66.

28. Checchia SL, Doneux PS, Miyazaki AN, Silva LA, Fregoneze M, Ossada A, et al. Biceps tenodesis associated with arthroscopic repair of rotator cuff tears. J Shoulder Elbow Surg. 2005;14(2):138-44.

29. Gartsman GM, O'Connor DP. Arthroscopic rotator cuff repair with and without arthroscopic subacromial decompression: a prospective, randomized study of one-year outcomes. J Shoulder Elbow Surg. 2004;13(4):424-6. 